\title{
A system to generate simultaneous forced oscillation and continuous positive airway pressure
}

\author{
R. Farré*, M. Rotger*, J.M. Montserrat**, D. Navajas*
}

A system to generate simultaneous forced oscillation and continuous positive airway pressure. R. Farré, M. Rotger, J.M. Montserrat, D. Navajas. CERS Journals Ltd 1997. ABSTRACT: Assessment of airway obstruction in patients with obstructive sleep apnoea (OSA) subjected to continuous positive airway pressure (CPAP) may be carried out using the forced oscillation technique (FOT). To facilitate routine application of forced oscillation (FO) in sleep studies, our aim was to design a system capable of generating CPAP and applying FOT simultaneously.

We constructed a prototype CPAP + FO generator by connecting a specially designed electromagnetic valve in parallel with a conventional blower. The capacity of the prototype to generate forced oscillation $(5 \mathrm{~Hz} \pm 1 \mathrm{hPa})$ was tested by connecting it to a model simulating spontaneous breathing.

The response of the prototype for target CPAPs of 5,10 and $15 \mathrm{hPa}$ and imposed sinusoidal breathing with peak flow up to $0.75 \mathrm{~L} \cdot \mathrm{s}^{-1}$ was excellent when compared with that reported for commercially available CPAP generators. The applicability of the prototype was tested by applying it to assess airway obstruction in four patients with OSA during sleep.

We conclude that the generator designed is able to apply continuous positive airway pressure and forced oscillation simultaneously. The system could be useful for automatic and noninvasive assessment of airway obstruction in patients with obstructive sleep apnoea subjected to continuous positive airway pressure. Future development of the generator may be helpful in implementing a set-up for automatic titration of continuous positive airway pressure. Eur Respir J 1997; 10: 1349-1353.
*Lab. Biofísica i Bioenginyeria and **Servei de Pneumologia (Hospital Clinic), Facultat de Medicina, Universitat de Barcelona, Spain.

Correspondence: R. Farré

Lab. Biofísica i Bioenginyeria

Facultat de Medicina

Casanova 143

E-08036 Barcelona

Spain

Keywords: Airway obstruction continuous positive airway pressure monitoring

respiratory impedance

respiratory resistance

sleep apnoea

Received: July 91996

Accepted after revision February 21997

This work was supported in part by Comisión Interministerial de Ciencia y Tecnología (SAF96-0076).
Assessment of airway obstruction in patients with obstructive sleep apnoea (OSA) is useful for the diagnosis and titration of the optimal continuous positive airway pressure (CPAP) [1]. In general, the most commonly used method to assess airway obstruction in patients with OSA is based on the recording of indirect signals (e.g. flow pattern, inductance plethysmograph, strain gauges, snoring, thermistors, etc.), which do not provide quantitative assessment of the degree of obstruction. Quantitative determination of the mechanical status of the upper airway requires the analysis of oesophageal pressure. However, measurement of this signal is invasive and is, therefore, of limited application in routine practice.

As has been suggested in recent studies in patients with OSA [2] and in models [3], the forced oscillation technique (FOT) may be a valuable tool for assessing the CPAP dependence of inspiratory and expiratory airway obstruction in sleep studies, since FOT is noninvasive, does not require patient co-operation, and provides automatic monitoring of airway resistance. The technique [4] consists of superimposing a low amplitude pressure oscillation on the CPAP level at the airway opening, and in computing respiratory resistance from the pressure and flow signals recorded at the nasal mask. To facilitate the routine application of FOT for diagnosis and titration of CPAP in patients with OSA, the aim of this work was to design and construct a prototype gen- erator capable of simultaneously applying CPAP and forced oscillation (FO) at the airway opening of spontaneously breathing subjects. The prototype was evaluated following established procedures $[5,6]$, and its applicability was assessed when used in patients with OSA subjected to different levels of CPAP during sleep.

\section{Methods}

The prototype constructed to simultaneously generate CPAP and FO at the airway opening was based on a specially designed shunt valve (fig. 1a), connected in parallel to a general purpose compressor-blower of reduced dimensions $(14 \times 14 \times 7.5 \mathrm{~cm})$ (model 117528-00 with controller 48063-00; Ametek, Technical Motor Division, Kent, $\mathrm{OH}, \mathrm{USA})$. For a given voltage driving the blower, pressure at the airway increases as the airflow resistance of the shunt valve rises, i.e. as the position of the piston in the shunt valve is displaced so as to close the airflow orifice. For each target CPAP, the blower was supplied with the constant voltage required to generate the desired CPAP with the airway closed, when the piston was at the central position of its excursion range. The means by which the device generated a target pressure (CPAP + FO), regardless of the external disturbances due to subject's breathing, was by modifying the degree of closure of the shunt valve with a servocontrolled system. The servocontrol modified the 
a)

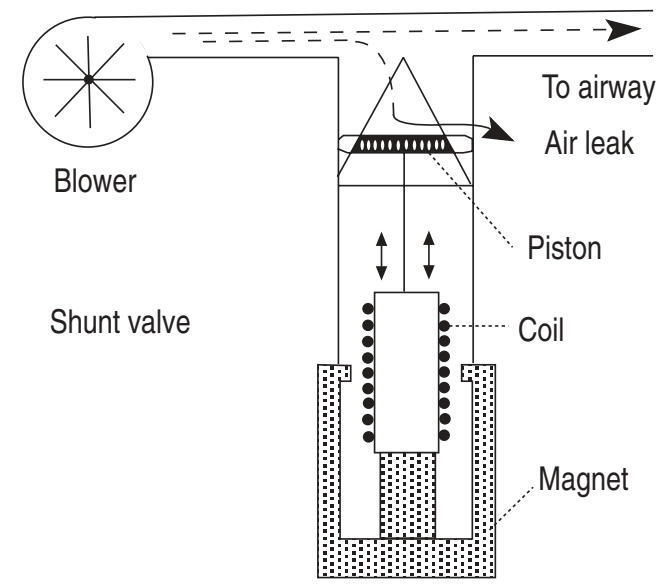

b)

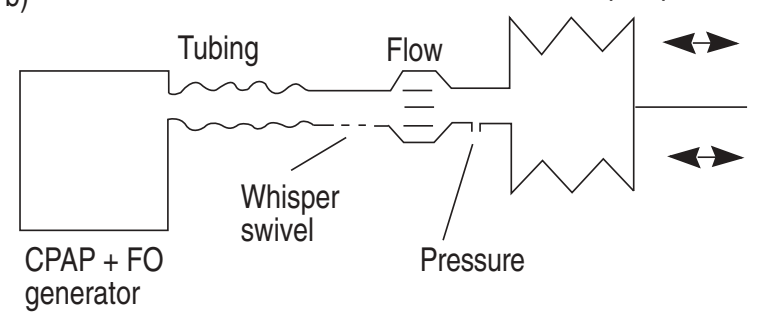

Fig. 1. - a) Diagram of the CPAP + FO generator. b) Experimental set-up for testing the prototype. CPAP: continuous positive airway pressure; FO: forced oscillation.

valve position to generate the desired target pressure at the airway opening. When the target pressure is constant, as it is in conventional CPAP application, the valve position is modified to compensate for the effects of the breathing flow, which tends to decrease or increase airway pressure during inspiration or expiration, respectively. When the target pressure consists of a constant value plus a forced oscillation, the servocontrol regulates the valve to apply CPAP + FO regardless of the breathing flow.

The position of the piston was modified by means of an electromagnetic actuator (fig. 1a) constructed with the magnet (12 cm diameter, $3 \mathrm{~cm}$ thick) of a conventional $250 \mathrm{~mm}$ woofer loudspeaker (Beyma, Valencia, Spain) and its modified coil. The coil was long enough to allow piston displacements of $20 \mathrm{~mm}$ within the linear range. To control the piston position, a conventional feedback loop was implemented. The coil was driven by a power amplifier fed with the output of a conventional proportional integral derivative (PID) controller, whose input was the difference between the target pressure (CPAP + FO) and the airway opening pressure recorded by means of a pressure transducer. In order to determine the servocontrol settings, attention was focused on the low frequency band (direct current (DC) $5 \mathrm{~Hz}$ ) including CPAP and the FO frequency of interest. Therefore, as described in detail previously [7], the coefficients of the electronic PID controller were set to achieve good steady-state and $5 \mathrm{~Hz}$ responses when the generator, connected to a resistance $\left(R=2.2 \mathrm{hPa} \cdot \mathrm{s} \cdot \mathrm{L}^{-1} ; 1 \mathrm{hPa}=1.02\right.$ $\left.\mathrm{cmH}_{2} \mathrm{O}\right)$ - inertance $\left(I=1.2 \mathrm{~Pa} \cdot \mathrm{s}^{2} \cdot \mathrm{L}^{-1}\right)$ - elastance $(E=18.2$ $\left.\mathrm{hPa} \cdot \mathrm{L}^{-1}\right)$ respiratory system analogue, was subjected to a step input.
In order to assess the performance of the prototype, we connected it to a system simulating a breathing patient. The set-up, shown in figure $1 \mathrm{~b}$, was similar to the ones employed to analyse the behaviour of commercially available CPAP devices $[5,6]$. Sinusoidal volume excursions were generated by a servocontrolled linear motor attached to rubber bellows [8]. As shown in figure $1 \mathrm{~b}$, the CPAP + FO prototype and the system simulating the breathing subject were connected by conventional silicone tubing $(150 \mathrm{~cm}$ in length, external diameter (ED) $22 \mathrm{~mm}$ ) and a whisper swivel. Pressure and flow at the airway opening were measured with a Honeywell 176PC transducer (Honeywell, Freeport, IL, USA), and a Fleisch No. II pneumotachograph (Metabo, Epalinges, Switzerland) with a Celesco pressure transducer $( \pm 2$ hPa) (Calesco, Canoga Park, CA, USA, respectively. Pressure and flow signals were analogically low-pass filtered (Butterworth, 8 poles, $8 \mathrm{~Hz}$ ), sampled at $256 \mathrm{~Hz}$ and stored in a computer. Volume was calculated by digital integration of flow.

According to the procedures described previously [5, $6]$, the performance of the prototype when acting as a CPAP ventilator (i.e. no FO superimposed) was tested for target CPAPs of 5,10 and $15 \mathrm{hPa}$, and simulated breathing of $0.5 \mathrm{~L}$ of tidal volume and inspiratory and expiratory peak flows of $0.5 \mathrm{~L} \cdot \mathrm{s}^{-1}\left(20\right.$ breaths $\left.\cdot \mathrm{min}^{-1}\right)$ or $0.75 \mathrm{~L} \cdot \mathrm{s}^{-1}$ (30 breaths $\left.\cdot \mathrm{min}^{-1}\right)$. Moreover, to test the response of the prototype when subjected to air leaks, we rapidly $(0.2 \mathrm{~s})$ imposed and maintained a flow leak of $1 \mathrm{~L} \cdot \mathrm{s}^{-1}$ at the airway opening, as described in detail previously [6]. The capability of the prototype to simultaneously generate CPAP and FO was assessed by targeting a FO with a frequency of $5 \mathrm{~Hz}$ and an amplitude of $\pm 1 \mathrm{hPa}$, and CPAPs of 5,10 and $15 \mathrm{hPa}$. These tests were also carried out with the simulated breathing described previously.

Moreover, to test its applicability for assessing airway obstruction, the CPAP + FO generator was applied to four male patients with OSA during sleep. To this end, the sinusoidal pump in figure 1a was replaced by a conventional nasal mask which was carefully fitted to minimize the shunt effect induced by possible air leaks. Airway obstruction was assessed from oscillatory resistance $(R)$ computed from the mask pressure and flow signals [3]. The study was carried out during a full polysomnography. During sleep, the prototype was used to modify the CPAP applied to the patient within a typical range $(5-15 \mathrm{hPa})$, and to simultaneously apply FO for monitoring airway obstruction.

\section{Results}

The performance of the CPAP + FO prototype when used as a conventional CPAP device is shown in figures $2 \mathrm{a}$ and $\mathrm{b}$, which plot the pressure actually generated at the airway opening when the simulated subject breathed at peak inspiratory rates of 0.5 and $0.75 \mathrm{~L} \cdot \mathrm{s}^{-1}$, respectively, for target CPAPs of 5,10 and $15 \mathrm{hPa}$. The recordings for $\mathrm{CPAP}=10 \mathrm{hPa}$ are accompanied by the ranges of actual pressure reported previously [5, 6] for different commercially available CPAP ventilators for identical testing conditions. Figure 2 shows pressure traces corresponding to three complete cycles of sinusoidal 

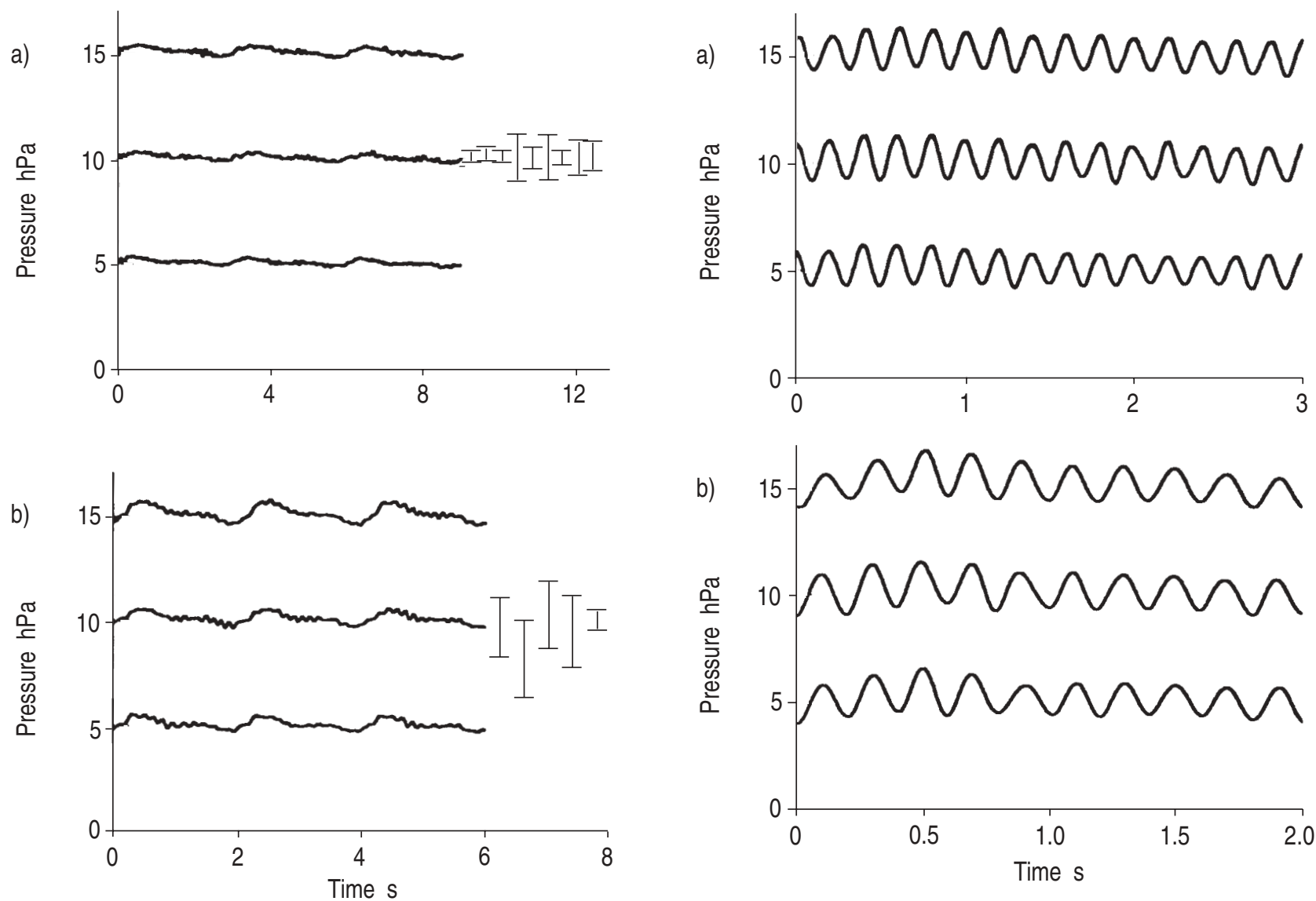

Fig. 2. - Airway pressure generated by the prototype for target CPAPs of 5, 10 and $15 \mathrm{hPa}$, when subjected to simulated sinusoidal breathing of $0.5 \mathrm{~L}$ of tidal volume and peak flows of: a) $0.5 \mathrm{~L} \cdot \mathrm{s}^{-1}$; and b) $0.75 \mathrm{~L} \cdot \mathrm{s}^{-1}$. The vertical bars correspond to the ranges of airway pressure found, respectively, in nine [5] and five [4] commercially available CPAP ventilators tested under the same conditions. CPAP: continuous positive airway pressure.

simulated breathing (periods of 3 and 2 s, respectively). As shown in this figure, the prototype performed as well as the best devices reported [5,6]. From the viewpoint of the simulated breathing subject connected to the CPAP + FO generator, the effective resistance of the device, which is not just that corresponding to the valve orifice but the result of the action of the whole servocontrolled system, was negligible $\left(0.75 \mathrm{hPa} \cdot \mathrm{s} \cdot \mathrm{L}^{-1}\right.$ at a CPAP of $15 \mathrm{hPa}$ and breathing peak flow of 0.75 $\mathrm{L} \cdot \mathrm{s}^{-1}$, which was the worst condition) (fig. $2 \mathrm{~b}$ ). When subjected to simulated sinusoidal breathing of $0.5 \mathrm{~L}$ of tidal volume and peak flow of $0.5 \mathrm{~L} \cdot \mathrm{s}^{-1}$, the workload that the prototype imposed on breathing was small (12, 17 and $22 \mathrm{~mJ} \cdot$ cycle $^{-1}$ at CPAPs of 5,10 and $15 \mathrm{hPa}$, respectively), when compared with the values reported previously [6] (ranging 30-120, 30-160, 30-200 $\mathrm{mJ} \cdot$ cycle $^{-1}$ at CPAPs of 5,10 and $15 \mathrm{hPa}$, respectively). The response of the prototype was also satisfactory compared with the values reported previously [6], when subjecting the generator to a leak of $1 \mathrm{~L} \cdot \mathrm{s}^{-1}$ at the airway opening for different CPAPs. Indeed, under such a leak, the airway pressure generated by the prototype was $4.5,10.0$ and $15.0 \mathrm{hPa}$ for target CPAPs of 5, 10 and $15 \mathrm{hPa}$, respectively, while the values reported previously [6] ranged $\sim 3.0-5.0, \sim 7.5-10.0$ and $\sim 12.5-14.9$ $\mathrm{hPa}$, respectively.

Fig. 3. - Airway pressure generated by the prototype when driven to produce superimposition of continuous positive airway pressure (CPAP) $(5,10$ and $15 \mathrm{hPa})$ and forced oscillation (FO) (2 hPa peakto-peak, $5 \mathrm{~Hz}$ ). The CPAP + FO generator was subjected to simulated sinusoidal breathing of $0.5 \mathrm{~L}$ of tidal volume and peak flows of: a) $0.5 \mathrm{~L} \cdot \mathrm{s}^{-1}$; b) $0.75 \mathrm{~L} \cdot \mathrm{s}^{-1}$.

The CPAP + FO prototype was able to generate the desired FO superimposed on the targeted CPAPs when applied to the simulated breathing subject. Figures 3a and $b$ show the airway pressure effectively generated at the different test conditions (peak flows of 0.5 and $0.75 \mathrm{~L} \cdot \mathrm{s}^{-1}$, respectively). These figures show pressure traces corresponding to a complete cycle of sinusoidal simulated breathing. Finally, figures 4 and 5 show representative examples of the practical performance of the $\mathrm{CPAP}+\mathrm{FO}$ generator when assessing airway obstruction in patients with OSA. These figures show the nasal flow $\left(V^{\prime} n\right)$, the pressure actually generated at the nasal mask $(P \mathrm{n})$, and the amplitude of the patient's oscillatory resistance $(R)$. As expected from the laboratory tests (figs. 2 and 3), the prototype was capable of generating the target pressure at the different flow conditions characterizing normal breathing, hypopnoeas and apnoeas at the different CPAP values. In the example presented in figure 4 , the patient was initially subjected to a high level of CPAP $(15 \mathrm{hPa})$, which was enough to normalize his breathing flow. Subsequently, the CPAP applied was reduced to a lower value $(6 \mathrm{hPa})$, and an obstructive apnoea appeared. Resistance $(R)$ was low and showed inspiratory and expiratory changes $[2,3]$ during normalized breathing at $\mathrm{CPAP}=15 \mathrm{hPa}$, and greatly increased during the obstructive apnoea at a 

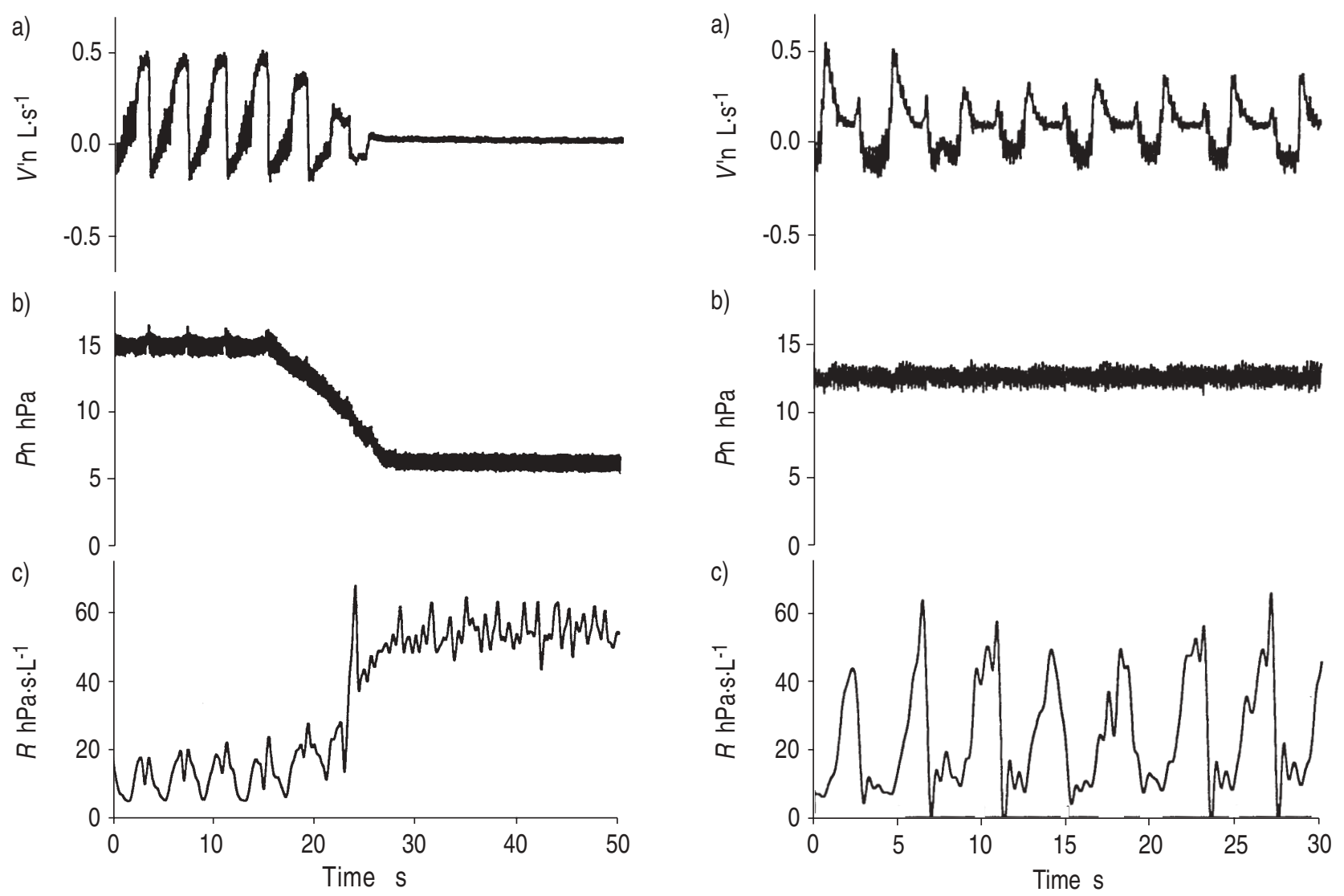

Fig. 4. - a) Nasal flow $\left(V^{\prime} n\right)$; b) pressure $\left(P_{n}\right)$; and c) oscillatory resistance $(\mathrm{R})$ measured in a patient with obstructive sleep apnoea (OSA) during sleep when the prototype was used to apply CPAP and FO simultaneously. The patient was initially subjected to a high level of CPAP (15 hPa), sufficient to normalize his breathing flow. Subsequently, the CPAP applied was reduced to a lower value $(6 \mathrm{hPa})$, and an obstructive apnoea appeared. $R$ was low and showed inspiratory and expiratory changes during normalized breathing at a CPAP of $15 \mathrm{hPa}$, and greatly increased during the obstructive apnoea at a CPAP of $6 \mathrm{hPa}$.

CPAP of $6 \mathrm{hPa}[2,3]$. In the example presented in figure 5, in which the same patient was subjected to a CPAP of $12 \mathrm{hPa}$, the inspiratory flow signal showed the characteristic pattern of obstructive hypopnoeas [9]. The marked phasic changes in $R$ showed that during these particular hypopnoeas the airway obstruction was mainly inspiratory [3].

\section{Discussion}

The prototype was able to simultaneously apply CPAP and FO within the ranges required for assessing airway obstruction during application of CPAP in spontaneously breathing patients. To test its performance, the generator underwent the same laboratory assays used recently by other authors $[5,6]$ to evaluate commercially available CPAP devices (fig. 2). Moreover, the applicability of the prototype was assessed, in patients with OSA during sleep, for continuous monitoring of airway obstruction along the different phases of the breathing cycle, even in the absence of flow (figs. 4 and $5)$. As FO was applied simultaneously with CPAP through a conventional mask, we observed that its applica-

Fig. 5. - a) Nasal flow $\left(V^{\prime} \mathrm{n}\right)$; b) pressure $\left(P_{\mathrm{n}}\right)$; and c) oscillatory resistance $(R)$ measured in a patient with obstructive sleep apnoea (OSA) during sleep when the prototype was used to apply CPAP and FO simultaneously. When the same patient as in the example presented in figure 4 was subjected to a CPAP of $12 \mathrm{hPa}$, the inspiratory flow signal showed the characteristic pattern of obstructive hypopnoeas. The marked phasic changes in $R$ showed that during these particular hypopnoeas the airway obstruction was mainly inspiratory. For definitions see legend to figure 1 .

tion did not impair the tolerance and compliance associated with conventional CPAP studies. Moreover, the use of the prototype did not modify the patient's sleep pattern and did not increase the number of arousals or microarousals.

Application of FOT requires the generation of a small pressure oscillation superimposed on the CPAP. To this end, a practical possibility could be to connect a FOT generator capable of withstanding pressure loads $[7,10]$ in parallel with a conventional CPAP ventilator. However, this procedure may conflict with the regulation systems incorporated in most CPAP ventilators [11]. Indeed, an ideal CPAP device may work so as to produce stable airway pressure regardless of external perturbations, such as the patient's breathing and, in this case, the oscillation of the FOT system. We designed a servocontrolled generator to simultaneously apply both the constant and the oscillatory pressure components at the airway, in order to avoid possible interferences when connecting independent CPAP and FOT devices.

The rationale of the prototype was based on placing a shunt valve in parallel with the blower, and not on servocontrolling the speed of the blower so as to impose 
the target pressure (CPAP + FO). This alternative, which is used in conventional CPAP devices to minimize the slow variations in airway pressure caused by the subject's breathing, proved to be inefficient in our application at $5 \mathrm{~Hz}$. Indeed, as the speed cannot easily be reversed in conventional blowers, and no braking functions are implemented, reduction in speed and, thus, in pressure is a passive and, consequently, slow process. By contrast, the use of an electromagnetic valve allowed us to modify the resistance of the shunt valve at the required rate. This approach was relatively simple to implement, since it is based on using a conventional blower and the magnet and rewired coil of a standard loudspeaker. In fact, after adequately modifying its electromechanical parameters, the servocontrolled valve designed could be adapted in order to use a conventional blower-based CPAP device as a flow source. The key feature of the shunt valve was that the large displacement range of the piston and the triangular profile of the air orifice almost linearized the relationship between piston position and airway pressure at each operating point, thereby optimizing the static and dynamic response of the servocontrolled CPAP + FO generator. The prototype could, potentially, be programmed to modify the value of CPAP applied, according to the degree of airway obstruction assessed by FOT. Such a procedure could be applicable for the implementation of a system for automatic CPAP titration and treatment.

In conclusion, the results obtained when testing the prototype in patients with obstructive sleep apnoea suggest that the device may be useful as a continuous positive airway pressure generator, as well as for automatic and noninvasive monitoring of airway obstruction during application of continuous positive airway pressure.

Acknowledgement: The authors wish to thank M.A. Rodríguez for his technical assistance.

\section{References}

1. Sullivan C, Issa F, Berthon-Jones M, Eves L. Reversal of obstructive sleep apnoea by continuous positive airway pressure applied through the nares. Lancet 1981; i: 862-865.

2. Navajas D, Farré R, Rotger M, Montserrat JM. Assessment of airway obstruction by means of the forced oscillation technique during application of CPAP in patients with SAS. Am J Respir Crit Care Med 1996; 153 (4): A772.

3. Farré R, Peslin R, Rotger M, Navajas D. Inspiratory dynamic obstruction detected by forced oscillation during CPAP: a model study. Am J Respir Crit Care Med 1997; (in press).

4. Peslin R, Fredberg JJ. Oscillation mechanics of the respiratory system. In: Handbook of Physiology. Vol 3. Part 1. Bethesda, MD, American Physiological Society, 1986; Section 3.

5. Jackson M, King MA, Shneerson JM. Intermachine variability in the stability of continuous positive airway pressure. Eur Respir J 1992; 5: 587-591.

6. Lofaso F, Heyer L, Leroy A, Lorino H, Harf A, Isabey D. Do turbines with servocontrolled speed improve continuous positive airway pressure generation? Eur Respir $J$ 1994; 7: 2077-2081.

7. Farré R, Ferrer M, Rotger M, Navajas D. Servocontrolled generator to measure respiratory impedance from 0.25 to $26 \mathrm{~Hz}$ in ventilated patients at different PEEP levels. Eur Respir J 1995; 8: 1222-1227.

8. Navajas D, Farré R. Respiratory input impedance during high frequency oscillatory ventilation. $\mathrm{Br} J$ Anaesth 1989; 63: 85S-90S.

9. Montserrat JM, Ballester E, Olivi H, et al. Time course of stepwise titration. Am J Respir Crit Care Med 1995; 152: 1854-1859.

10. Peslin R, Felicio da Silva J, Duvivier C, Chabot F. Respiratory mechanics studied by forced oscillations during artificial ventilation. Eur Respir J 1993; 6: 772-784.

11. Kacmarek RM, Hess D. Equipment required for home mechanical ventilation. In: Tobin MJ, ed. Principles and Practice of Mechanical Ventilation. New York, McGrawHill, 1994; pp. 111-154. 